\title{
大环化合物的合成、结构及其对镧(III)离子的识别研究
}

\author{
欧 敏 ${ }^{a}$ 邓雅欣 ${ }^{a}$ 王芳芳 ${ }^{a}$ 朱 纯 ${ }^{a}$

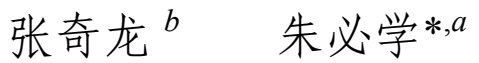 \\ ( 贵州大学大环及超分子化学重点实验室 贵阳 550025) \\ ( ${ }^{b}$ 贵阳医学院化学教研室 贵阳 550004)
}

\begin{abstract}
摘要 在 $\mathrm{Ba}^{2+}$ 离子诱导作用下, 采用前体二醛 $\mathbf{1}$ [1,2-双(2'-甲酰苯氧基)乙烷]和前体二胺 2 [间苯二酚-双(4-胺基苯基)醚] 进行缩合, 得到 $[1+1]$ Schiff 碱大环化合物 $\mathbf{3}$, 进一步将大环 $\mathbf{3}$ 还原得大环 $\mathbf{4}$, 用 ${ }^{1} \mathrm{H} \mathrm{NMR}$, IR, 质谱和元素分析等对其组 成进行了表征. 采用 X 射线衍射技术测定了大环 $\mathbf{3}$ 和相应的还原产物 $\mathbf{4}$ 的晶体结构. 结构解析结果表明, 大环 $\mathbf{3}$ 的分 子呈折叠结构, 4 表现为扭曲的非叠结构. 采用 UV-vis 光谱技术, 对大环与系列稀土离子的键合作用进行了考察, 结果 表明, 大环化合物 $\mathbf{3}$ 对锞(III)离子具有选择性识别作用. 进一步采用 Job 法确定了大环 $\mathbf{3}$ 与锞(III)离子配位作用的键合 比为 $1: 1$, 键合常数为 $7.59 \times 10^{3}$.
\end{abstract}

关键词 大环化合物; 晶体结构; 镧(III)离子; 识别

\section{Synthesis, Structures of Macrocyclic Compounds and Study on Recognition for La(III) Ion}

\author{
$\mathrm{Ou}, \operatorname{Min}^{a}$ \\ Deng, Yaxin ${ }^{a}$ \\ Wang, Fangfang ${ }^{a}$ \\ Zhu, Chun ${ }^{a}$ \\ Zhang, Qilong ${ }^{b}$ \\ Zhu, Bixue ${ }^{*, a}$ \\ $\left({ }^{a}\right.$ Key Laboratory of Macrocyclic and Supramolecular Chemistry, Guizhou University, Guiyang 550025) \\ ( ${ }^{b}$ Department of Chemistry, Guiyang Medical College, Guiyang 550004)
}

\begin{abstract}
Schiff base macrocyclic compound 3 has been synthesized from precursor 1 [1,2-bis(2'-formacylphenoxy)ethane] and precursor 2 [2-resorcinol-bis(4-aminophenyl)ether] via condensation and cyclization using $\mathrm{Ba}^{2+}$ as template. The macrocycle 3 was further reduced giving macrocyclic compound $\mathbf{4}$, and the structures were characterized by ${ }^{1} \mathrm{H}$ NMR, IR, MS and elemental analysis, respectively. The X-ray single crystal analysis reveals that the macrocycle 3 has a folding structure, and $\mathbf{4}$ shows a twisted unfolding structure. The study for the reaction of the macrocyclic compound $\mathbf{3}$ or $\mathbf{4}$ with a series of the rare earth ions is performed by UV-Vis absorption spectra technique, and the results show that the macrocycle $\mathbf{3}$ displayed a selective recognition for $\mathrm{La}^{3+}$ ion. Moreover, the JOB plot exhibited a $1: 1$ (host $:$ guest) stoichiometry between the host 3 and $\mathrm{La}^{3+}$, and the binding constant $(K)$ was determined using UV-vis titration method, $K=7.59 \times 10^{3}$.

Keywords macrocyclic compound; crystal structure; La(III) ion; recognition
\end{abstract}

大环化合物在分子识别、主客体化学、磁性材料、 催化化学等方面的应用越来越广泛 ${ }^{[1,2]}$, 设计和合成新 型的大环分子是目前大环化学及超分子化学研究的热 点 ${ }^{[3]}$. 在众多的大环化合物中, 含氮、氧等杂原子的大环 配体具有环腔大小可控、环内配位供体原子(氮、氧原 子)数目可变 $[4,5]$, 特别是大环结构刚、柔性易于调整，使 得类似大环在同金属离子进行配位键合作用时，一方面
因为环缘上的氮、氧原子配位取向灵活多变, 易于得到 新颖的配合物结构 ${ }^{[6,7]}$; 另一方面, 有利于提高其与金属 离子配位作用的选择性 ${ }^{[8]}$. 因此设计、合成含氮、氧等 杂原子的有机大环, 并开展其与金属离子之间的配位化 学研究一直受到人们的普遍青睐 ${ }^{[9]}$.

据此，本文先合成前体二醛 1 [1,2-双(2'-甲酰苯氧 基)乙烷]和二胺 2 [间苯二酚-双(4-氨基苯基)醚]，经缩

*E-mail: sci.bxzhu@gzu.edu.cn

Received January 24, 2013; revised March 15, 2013; published online April 3, 2013.

Project supported by the National Natural Science Foundation of China (No. 21061003) and the Natural Science Foundation of Guizhou Province (No. [2012]2151).

国家自然科学基金(No. 21061003)、贵州省自然科学基金(No. [2012]2151)资助项目. 
合得到 [1+1] Schiff 碱大环化合物 $\mathbf{3}$, 进一步将 Schiff 碱 3 还原得到柔性较好的大环 4 . 同时, 采用 $\mathrm{X}$ 单晶衍 射技术这 2 个大环化合物的晶体进行了结构解析, 并分 别考察了这 2 个大环与系列稀土离子的配位作用性质. 大环化合物合成路线见 Scheme 1.
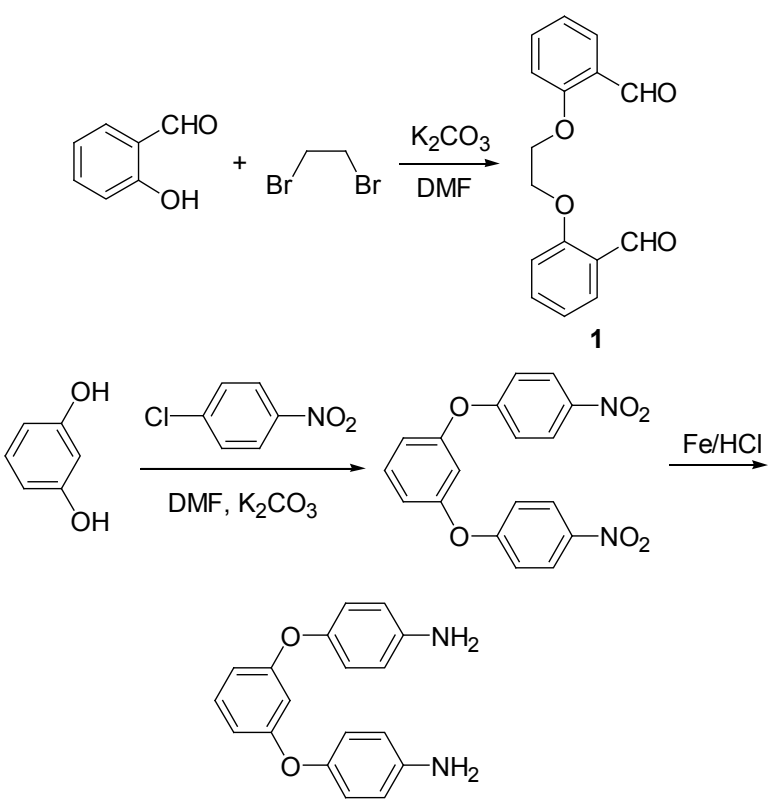

2
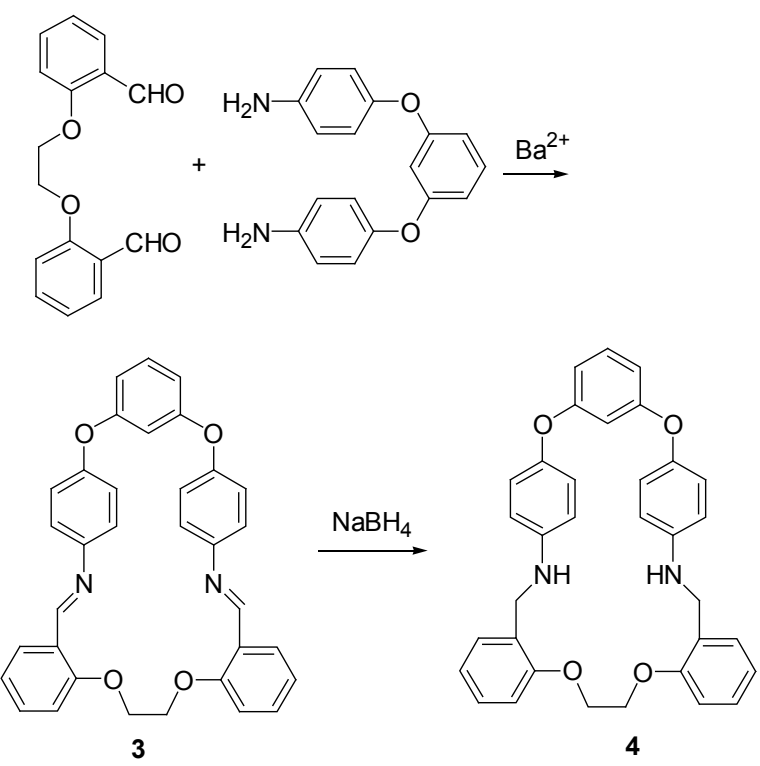

Scheme 1

\section{1 实验部分}

\section{1 仪器和试剂}

Bio-Rad 型傅立叶红外光谱仪 ( $4000 \sim 400 \mathrm{~cm}^{-1}$ ); Ry-2 型熔点仪(温度计未校正); JEOL ECX $400 \mathrm{MHz}$ 核 磁共振仪; Vario EL III 型元素分析仪(德国); 岛津 UV-1800 紫外分光光度计(日本); MS (Angilent 1100)质
谱仪; 晶体结构测定采用 Bruker Smart Apex 衍射仪.

间苯二酚, 对硝基氯苯(阿拉丁化学试剂有限公司), 水杨醛，1,2-二溴乙烷，水合肼 $(80 \%)$ (天津市科密欧化学 试剂有限公司), 其他试剂均为市售分析纯.

\section{2 合成}

\subsection{1 前体二醛 $\mathbf{1}$ 的合成}

按照类似于文献[10]的方法，从 2-羟基苯甲醛和 1,2-二溴乙烷制得前体二醛，1,2-二(2'-甲酰苯氧基)乙烷 (1)为淡黄色固体, 产率 71.6\%. m.p. $129 \sim 131{ }^{\circ} \mathrm{C} ;{ }^{1} \mathrm{H}$ NMR $\left(\mathrm{CDCl}_{3}, 400 \mathrm{MHz}\right) \delta$ : 10.45 (s, 2H, CHO), 7.85 7.87 (m, 2H, Ar-H), $7.57 \sim 7.61$ (m, 2H, Ar-H), 7.06 7.11 (m, 4H, Ar-H), 4.54 (s, 2H, $\left.\mathrm{CH}_{2}\right)$.

\subsection{2 前体二胺 2 的合成}

以间苯二酚，对硝基氯苯为原料，按文献[11]的方 法合成化合物间苯二酚二(4-硝基苯基)醚, 进一步还原 得间苯二酚二(4-氨基苯基)醚(2)为浅黄色固体，经真空 干燥、称量, 产率为 $65 \%$. m.p. $136 \sim 138{ }^{\circ} \mathrm{C} ;{ }^{1} \mathrm{H}$ NMR $\left(\mathrm{CDCl}_{3}, 400 \mathrm{MHz}\right) \delta: 7.14(\mathrm{t}, J=8 \mathrm{~Hz}, 1 \mathrm{H}, \mathrm{ArH}), 6.85 \sim$ $6.88(\mathrm{~m}, 4 \mathrm{H}, \mathrm{ArH}), 6.65 \sim 6.69(\mathrm{~m}, 4 \mathrm{H}, \mathrm{ArH}), 6.54(\mathrm{t}, J=4$ $\mathrm{Hz}, 3 \mathrm{H}, \mathrm{ArH}), 3.58$ (s, 2H, NH $\left.\mathrm{N}_{2}\right), 1.65\left(\mathrm{~s}, 2 \mathrm{H}, \mathrm{NH}_{2}\right)$.

\subsubsection{Schiff 碱大环化合物 3 的合成}

称取 $0.146 \mathrm{~g}(0.5 \mathrm{mmol}) 2$ 和 $\mathrm{BaCl}_{2} \cdot \mathrm{H}_{2} \mathrm{O}(0.122 \mathrm{~g})$ 于 $250 \mathrm{~mL}$ 圆底烧瓶中, 加入 $50 \mathrm{~mL}$ 的无水甲醇, 搅拌至溶 解. 向混合液中, 缓慢滴加二醛 $\mathbf{1}(0.137 \mathrm{~g}, 0.5 \mathrm{mmol})$ 的 无水甲醇溶液 $(50 \mathrm{~mL})$. 溶液逐渐变浑浊并有淡黄色沉 淀生成, 室温下继续摚拌 $10 \mathrm{~h}$. 旋蒸除去溶剂, 过滤, 滤饼依次用无水甲醇洗涤 3 次，乙醚洗涤 2 次，真空干 燥得大环 3 的淡黄色固体粉末 $0.21 \mathrm{~g}$, 产率 75.7\%. m.p. $272 \sim 274{ }^{\circ} \mathrm{C} ;{ }^{1} \mathrm{H}$ NMR (DMSO- $\left.d_{6}, 400 \mathrm{MHz}\right) \delta: 8.80$ (s, $2 \mathrm{H}, \mathrm{CH}=\mathrm{N}), 7.97 \sim 7.98(\mathrm{~m}, 2 \mathrm{H}, \operatorname{ArH}), 7.44 \sim 7.52(\mathrm{~m}$, $3 \mathrm{H}, \mathrm{ArH}), 7.18(\mathrm{~d}, J=8 \mathrm{~Hz}, 6 \mathrm{H}, \mathrm{ArH}), 7.01 \sim 7.09$ (m, 6H, ArH), $6.94 \sim 6.96(\mathrm{~m}, 2 \mathrm{H}, \mathrm{ArH}), 6.14(\mathrm{t}, J=2.5 \mathrm{~Hz}, 1 \mathrm{H}$, $\mathrm{ArH}), 4.58$ (s, 4H, $\left.\mathrm{OCH}_{2}\right)$; IR (KBr) v: $2927\left(\mathrm{CH}_{2}\right), 1621$ $(\mathrm{C}=\mathrm{N}), 1582,1483,1499(\mathrm{C}=\mathrm{C}), 1285(\mathrm{C}-\mathrm{O}) \mathrm{cm}^{-1}$; FABMS $m / z$ : $549[\mathrm{M}+\mathrm{Na}]^{+}$. Anal. calcd for $\mathrm{C}_{34} \mathrm{H}_{26} \mathrm{~N}_{2} \mathrm{O}_{4}$ : C 77.55, H 4.98, N 5.32; found C 77.57, H 4.94, N 5.31.

取少量淡黄色固体 3 溶解于 $\mathrm{CH}_{3} \mathrm{OH}$ 和 $\mathrm{CHCl}_{3}(\mathrm{~V}$ : $V=1: 1)$ 的混合溶剂中, $3 \mathrm{~d}$ 后长出适合 $\mathrm{X}$ 射线单晶衍射 测定的黄色块状晶体.

\subsection{4 大环化合物 4 的合成}

称取 $0.8 \mathrm{~g}$ 的 Schiff 碱大环化合物 $\mathbf{3}$, 溶解于 $30 \mathrm{~mL}$ 四氢呋喃中, 加入 $0.3 \mathrm{~g}$ 钯碳, 然后缓慢滴加 $0.15 \mathrm{~g}$ $\mathrm{NaBH}_{4}$ 的乙醇溶液 $(10 \mathrm{~mL})$, 回流反应 $24 \mathrm{~h}$. 旋去溶剂, 加入 $30 \mathrm{~mL}$ 蒸馏水, 用 $\mathrm{CH}_{2} \mathrm{Cl}_{2}$ 萃取 $2 \sim 3$ 次, 旋蒸除去 
$\mathrm{CH}_{2} \mathrm{Cl}_{2}$, 真空干燥、得大环 4 的黄色固体粉末 $0.41 \mathrm{~g}$, 产 率 49\%. m.p. 90 92 ${ }^{\circ} \mathrm{C}$; ${ }^{1} \mathrm{HNMR}\left(\mathrm{CDCl}_{3}, 400 \mathrm{MHz}\right) \delta$ : $7.38(\mathrm{~d}, J=6.8 \mathrm{~Hz}, 2 \mathrm{H}, \mathrm{ArH}), 6.93 \sim 6.99$ (m, 5H, ArH), $6.73 \sim 6.79(\mathrm{~m}, 8 \mathrm{H}, \mathrm{ArH}), 6.49 \sim 6.52(\mathrm{~m}, 4 \mathrm{H}, \mathrm{ArH}), 6.04$ (t, $J=2.5 \mathrm{~Hz}, 1 \mathrm{H}, \mathrm{ArH}), 4.44\left(\mathrm{~s}, 4 \mathrm{H}, \mathrm{OCH}_{2} \mathrm{CH}_{2} \mathrm{O}\right), 4.32$ (s, $4 \mathrm{H}, \mathrm{N}-\mathrm{CH}_{2}$ ), 3.49 (s, H, NH), 2.62 (s, H, NH); IR (KBr) v: $3429(\mathrm{~N}-\mathrm{H}), 3040(\mathrm{Ar}-\mathrm{H}), 2926\left(\mathrm{CH}_{2}\right), 1448,1509$, $1600(\mathrm{C}=\mathrm{C}), 1168(\mathrm{C}-\mathrm{N})$; MS (ESI) $m / z: 531\left(\mathrm{M}^{+}+\mathrm{H}\right)$. Anal. calcd for $\mathrm{C}_{34} \mathrm{H}_{30} \mathrm{~N}_{2} \mathrm{O}_{4}$ : C 76.96, H 5.70, N 5.28; found C 76.98, H 5.66, N 5.29.

将少量制得的黄色固体溶解于 $\mathrm{CH}_{3} \mathrm{OH}$ 和 $\mathrm{CH}_{2} \mathrm{Cl}_{2}$ $(V: V=1: 2)$ 的混合溶剂中, $5 \mathrm{~d}$ 后长出适合 $\mathrm{X}$ 射线单晶 衍射测定的黄色块状晶.

\section{3 晶体结构测定}

选取大小适当的晶体, 用 Bruker Smart Apex 单晶 衍射仪, 采用经石墨单色器单色化的 Mo $\mathrm{K} \alpha$ 射线 $(\lambda=$ $0.071073 \mathrm{~nm})$, 以 $\varphi-\omega$ 扫描方式收集单晶衍射数据. 强 度数据进行了经验吸收校正、 LP 校正. 晶体结构由直接 法解得. 对全部非氢原子坐标及其各向异性热参数进行 了全矩阵最小二乘法修正. 所有计算用 SHELX-97 程序 完成 ${ }^{[12]}$ (有关晶体学数据详见辅助材料表 S1).

\section{4 离子识别试验}

分别移取一定体积、浓度为 $1 \times 10^{-4} \mathrm{~mol} \cdot \mathrm{L}^{-1}$ 的大 环 3 或 4 的 $\mathrm{CH}_{3} \mathrm{CN}$ 溶液于一系列 $10 \mathrm{~mL}$ 的容量瓶中, 分 别加入一定浓度、不同体积的系列稀土离子(即 $\mathrm{La}^{3+}$, $\mathrm{Ce}^{3+}, \mathrm{Pr}^{3+}, \mathrm{Nd}^{3+}, \mathrm{Sm}^{3+}, \mathrm{Eu}^{3+}, \mathrm{Tb}^{3+}, \mathrm{Dy}^{3+}, \mathrm{Er}^{3+}, \mathrm{Tm}^{3+}$, $\mathrm{Yb}^{3+}, \mathrm{Lu}^{3+}$ )的硝酸盐的乙腈溶液, 用 $\mathrm{CH}_{3} \mathrm{CN}$ 稀释至刻 度, 混合均匀、放置达体系吸光度稳定不变后, 室温下, 在 $200 \sim 600 \mathrm{~nm}$ 范围内记录各反应体系的 UV-vis 光谱 $\left(\mathrm{CH}_{3} \mathrm{CN}\right.$ 作参比).

\section{2 结果与讨论}

在合成得到 Schiff 碱大环化合物 3 和相应的还原 产物 4 基础上, 通过单晶培养和晶体结构解析, 进一步 获得大环 3 和 4 的晶体结构数据. 大环 3 和 4 的晶体学 及结构修正数据见辅助材料表 $\mathrm{S} 1$, 选择性键长和键角 数据见辅助材料表 $S 2$. 大环化合物 3 和 4 晶体结构数据 存于英国剑桥数据中心, CCDC: 920469, 3; 920470, 4 .

\section{1 大环 3 和 4 的晶体结构描述}

Schiff 碱大环 3 和大环化合物 $\mathbf{4}$ 的分子结构如图 1. 在 $[1+1]$ Schiff 碱大环化合物 3 分子结构中(图 1), 在间 苯二酚-双(4-胺基苯基)醚结构单元中，位于中央的苯环 与位于其两臂, 经氧桥连的两个苯环之间的二面角分别 为 $124^{\circ}$ 和 $106.7^{\circ}$, 处在两臂的这两个苯环之间的二面角
为 $117^{\circ}$. 位于大环两个侧链, 经 Schiff 碱 $\mathrm{C}=\mathrm{N}$ 双键连 接的两对苯环之间的二面角分别为 $101^{\circ}$ 和 $127.4^{\circ}$; 位于 大环下缘, 由烷基链连接的两个苯环之间的二面角为 $154.7^{\circ}$ 和 $118.9^{\circ}$, 以间苯二酚中央苯环 $\mathrm{C}(2)$ 和 $\mathrm{C}(5)$ 所连 接的直线为轴, 大环分子呈一折叠结构(如图 $2 \mathrm{a}$ ). 分子 中 Schiff 碱碳氮双键 $\mathrm{C}(13)=\mathrm{N}(1)$ 和 $\mathrm{C}(28)=\mathrm{N}(2)$ 键长分 别为 0.1264(5)和 0.1260(5) $\mathrm{nm}$ (见辅助材料, 表 S2), 是 典型的碳氮双键的建长 (典型的 $\mathrm{C}=\mathrm{N}$ 双键的平均键长 为 $0.126 \mathrm{~nm})^{[13]}$.

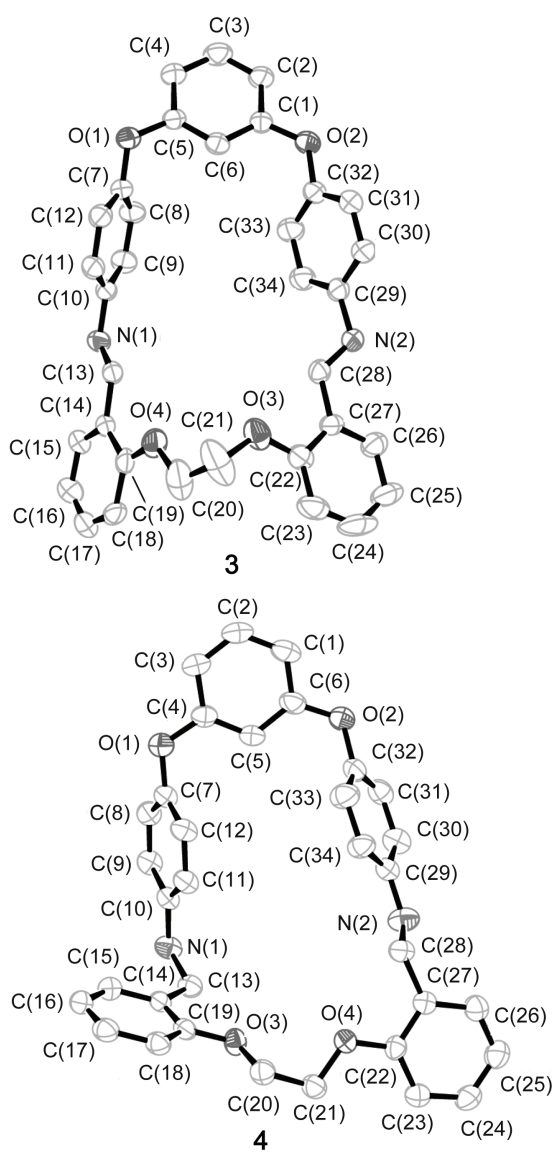

图 1 大环化合物 3 和 4 的分子结构(椭球几率为 30\%)

Figure 1 Molecular structures of the macrocycle 3 and 4 (probability of ellipsoid is $30 \%$ )

在还原产物 4 的结构中(图 1), 碳氮单键 $\mathrm{C}(13)-$ $\mathrm{N}(1)$ 和 $\mathrm{C}(28)-\mathrm{N}(2)$ 键长分别为 $0.1442(3)$ 和 0.1403 (3) $\mathrm{nm}$, 在间苯二酚二(4-胺基苯基)醚结构单元部分，位于 中央的苯环与位于其两臂，经氧桥连的两个苯环之间的 二面角分别为 $113.65^{\circ}$ 和 $112.65^{\circ}$, 处在其两臂的这两个 苯环之间的二面角为 $127.68^{\circ}$. 位于大环两个侧链, 经 Schiff 碱 $\mathrm{C}-\mathrm{N}$ 键连接的两对苯环之间的二面角分别为 $125.66^{\circ}$ 和 $101.68^{\circ}$; 位于大环下缘, 由烷基链连接的两 个苯环之间的二面角为 $112.28^{\circ}$. 从图 $2 \mathrm{~b}$ 可见，相对于 大环 3 而言，大环 4 为扭曲的非叠结构，这应源于 Schiff 


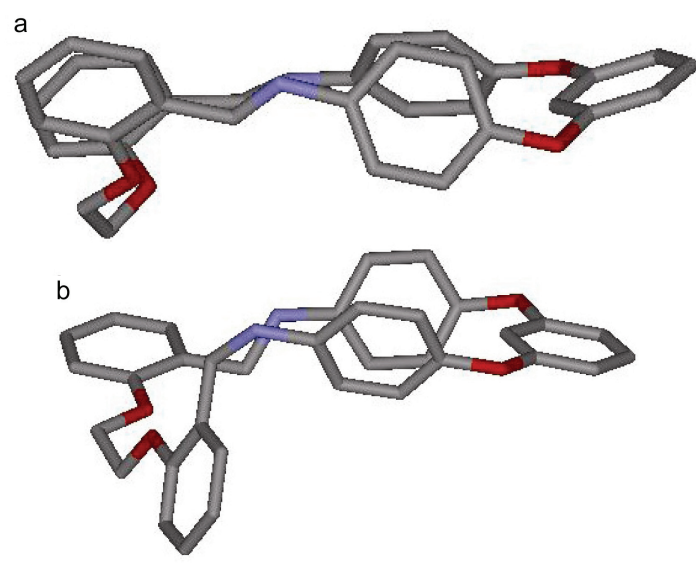

图 2 (a)大环化合物 3 的折叠结构及(b) 4 的扭曲结构

Figure 2 (a) The enfold structure of the macrocycle 3 and (b) the twisty structure of $\mathbf{4}$

碱大环 3 中 Schiff 碱 $\mathrm{C}=\mathrm{N}$ 双键, 在大环 4 中被还原为 $\mathrm{C}-\mathrm{N}$ 单键后，分子结构柔性增加所致.

大环 3 和 4 相比, 在大环 3 中由于 Schiff 碱 $\mathrm{C}=\mathrm{N}$ 的存在, 其中央苯环的两个酚氧原子 $[\mathrm{O}(1)$ 和 $\mathrm{O}(2)]$ 之间 的距离为 $0.4852 \mathrm{~nm}$, 位于两侧链的 $\mathrm{N}(1)$ 和 $\mathrm{N}$ (2) 原子之 间的距离为 $0.6974 \mathrm{~nm}$. 在大环 4 中, 相应的中央苯环的 两个酚氧原子 $[\mathrm{O}(1)$ 和 $\mathrm{O}(2)]$ 之间的距离为 $0.4838 \mathrm{~nm}$, 位 于两侧链的 $\mathrm{N}(1)$ 和 $\mathrm{N}(2)$ 原子之间的距离为 $0.6548 \mathrm{~nm}$, 即大环 4 中两个氮配位供体原子间距进一步变小. 这是 由于大环 3 中的 $\mathrm{C}=\mathrm{N}$ 双键被还原为大环 4 中的 $\mathrm{C}-\mathrm{N}$ 单键，大环 4 比 3 具有更大的柔性所致.

\section{2 大环与稀土金属离子配位作用考察}

2.2.1 大环与系列稀土离子配位反应体系的 UV-vis 光谱

分别移取 $1 \mathrm{~mL}$ 浓度为 $1 \times 10^{-4} \mathrm{~mol} \cdot \mathrm{L}^{-1}$ 的大环 3 和 4 的 $\mathrm{CH}_{3} \mathrm{CN}$ 溶液于一系列 $10 \mathrm{~mL}$ 容量瓶中, 分别加入浓 度均为 $1 \times 10^{-3} \mathrm{~mol} \cdot \mathrm{L}^{-1}$ 的系列稀土离子(即 $\mathrm{La}^{3+}, \mathrm{Ce}^{3+}$, $\mathrm{Pr}^{3+}, \mathrm{Nd}^{3+}, \mathrm{Sm}^{3+}, \mathrm{Eu}^{3+}, \mathrm{Tb}^{3+}, \mathrm{Dy}^{3+}, \mathrm{Er}^{3+}, \mathrm{Tm}^{3+}, \mathrm{Yb}^{3+}$, $\mathrm{Lu}^{3+}$ ) 等金属离子的硝酸盐的 $\mathrm{CH}_{3} \mathrm{CN}$ 溶液 $0.5 \mathrm{~mL}$, 用 $\mathrm{CH}_{3} \mathrm{CN}$ 稀释至刻度, 混合均匀, 放置达体系吸光度稳定 不变后, 在室温下, 记录得到各反应体系的紫外-可见 吸收光谱如图 $3\left(\mathrm{CH}_{3} \mathrm{CN}\right.$ 作参比).

从图 3 可见, 自由配体 Schiff 碱大环 $\mathbf{3}$ 和 $\mathbf{4}$ 的紫外 最大吸收 $\lambda_{\text {max }}$ 分别在 328 和 $312 \mathrm{~nm}$ 处. 结果表明, 在所 考察的系列稀土离子中, 大环 4 对所考察的系列稀土离 子均未能观察到明显作用(即大环配体的 $\lambda_{\text {max }}$ 几乎没变 化), 仅大环 3 在加入金属 $\mathrm{La}^{3+}$ 离子后, 体系的 $\lambda_{\text {max }}$ 从 $328 \mathrm{~nm}$ 蓝移到 $312 \mathrm{~nm}$ 处. 在所考察的系列稀土离子中, $\mathrm{La}^{3+}$ 相对具有较大的离子半径, 大环 3 对 $\mathrm{La}^{3+}$ 表现出明 显的选择性键合作用, 表明大环 3 与 $\mathrm{La}^{3+}$ 键合时, 3 在环
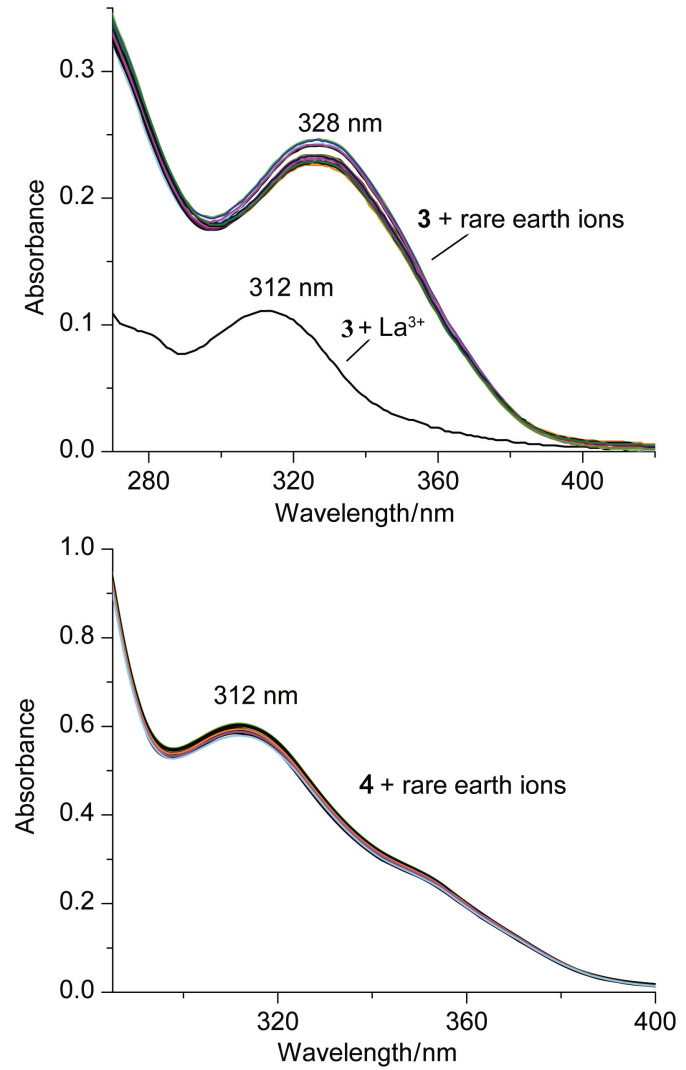

图 3 稀土金属离子存在时 Schiff 碱大环 $\mathbf{3}$ 和大环 $\mathbf{4}$ 的 UV-vis 吸收光谱

Figure 3 UV-vis absorption spectra of macrocycles 3 and $\mathbf{4}$ in the presence of various rare earth ions

腔尺寸以及环缘上配位原子取向等方面存在着较好的 同 $\mathrm{La}^{3+}$ 配位的匹配关系. 在此基础上，进一步测定了大 环 3 与金属 $\mathrm{La}^{3+}$ 离子配位作用的配位比及配位稳定常 数.

2.2.2 Schiff 碱大环 3 与 $\mathrm{La}^{3+}$ 离子配位反应的配位比 及稳定常数

Schiff 碱大环 3 与 $\mathrm{La}^{3+}$ 离子的 UV-vis 光谱滴定: 室 温下, 分别移取 $1 \mathrm{~mL}$ 浓度为 $2.00 \times 10^{-4} \mathrm{~mol} \cdot \mathrm{L}^{-1}$ 的大环 3 的乙腈溶液, 加入系列 $10 \mathrm{~mL}$ 的容量瓶中, 向其中分 别滴加浓度为 $2.00 \times 10^{-4} \mathrm{~mol} \cdot \mathrm{L}^{-1}$ 、不同体积的 $\mathrm{La}^{3+}$ 硝 酸盐的乙腈溶液, 以乙腈溶剂定容, 得到 $\mathrm{La}^{3+}$ 浓度依次 增加的系列反应体系. 待反应达平衡后，在 $200 \sim 600$ $\mathrm{nm}$ 范围内进行 UV-vis 光谱扫描(如图 4). 从图 4 可见, 随着体系中 $\mathrm{La}^{3+}$ 浓度增加, 体系的吸光度在 $328 \mathrm{~nm}$ 处 依次下降, 曲线呈现两个等吸收点.

配位比确定: 首先采用摩尔比法测定了配位体系的 键合比. 实验中用选择性较高的 $328 \mathrm{~nm}$ 波长处, 各作用 体系吸光度 $A$ 对大环与 $\mathrm{La}^{3+}$ 的摩尔比 $\left(n_{3} / n_{\mathrm{La}^{3+}}\right)$ 作图(如图 5a). 可见, 当大环 3 与 $\mathrm{La}^{3+}$ 的物质的量比为 $1: 1$ 时, 体 系吸光度趋向平缓并出现转折, 表明在溶液中, 3 与 


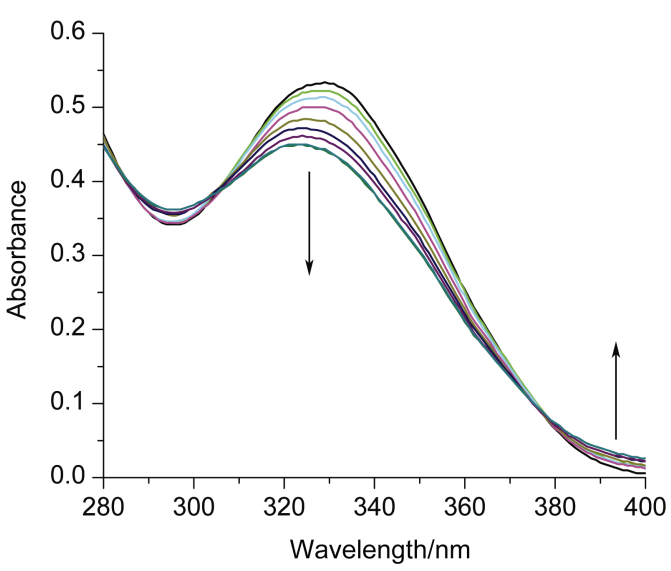

图 4 大环 3 与 $\mathrm{La}^{3+}$ 反应体系的 UV-vis 吸收光谱滴定曲线 Figure 4 The titration curve of UV-vis absorption spectrum for the system involving macrocycle 3 with $\mathrm{La}^{3+}$

$\mathrm{La}^{3+}$ 形成了 $1: 1$ 的配合物.
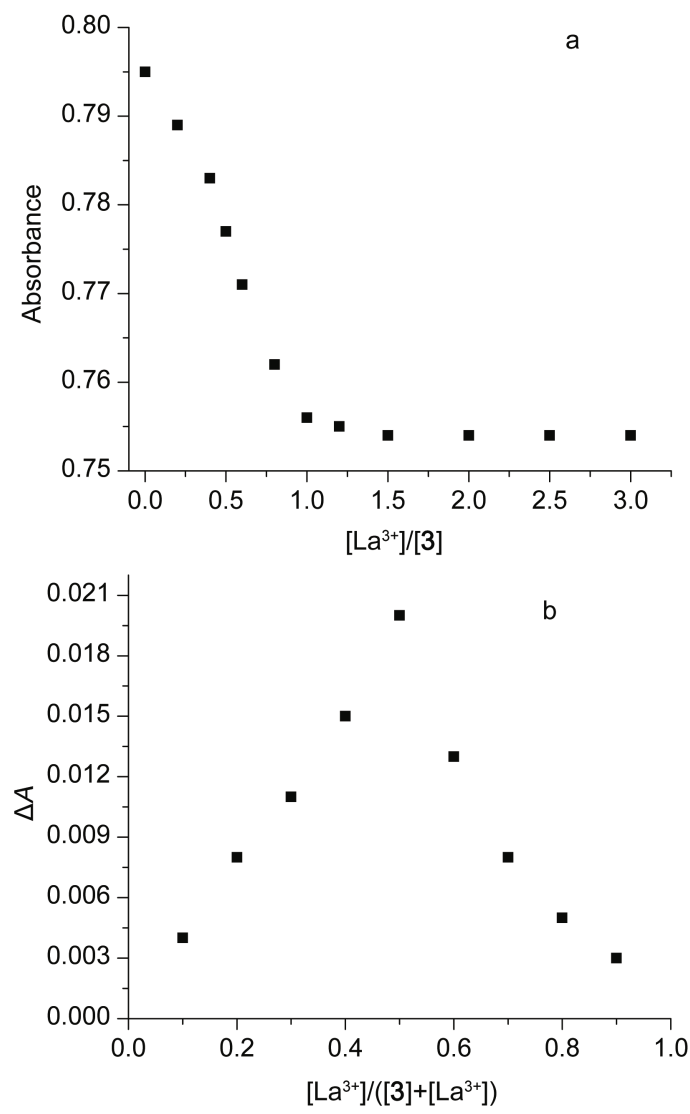

图 5 (a)大环 3 与 $\mathrm{La}^{3+}$ 配位反应体系的 $\mathrm{A}$ 与 $\left[\mathrm{La}^{3+}\right] /[3]$ 的关系 和(b) Jo 图

Figure 5 (a) Plot of $\mathbf{A} v s$. $\left[\mathrm{La}^{3+}\right] /[\mathbf{3}]$ for the system involving macrocycle 3 with $\mathrm{La}^{3+}$ and Job plot (b)

同时，采用了 Job 法进一步确定体系配位作用的键 合比，即在乙腈溶液中，分别配置浓度均为 $4.00 \times 10^{-4}$ $\mathrm{mol} \cdot \mathrm{L}^{-1}$ 大环 3 和 $\mathrm{La}^{3+}$ 离子的乙腈溶液作为操作液. 固 定各配位反应体系中，反应物总浓度为 $4.00 \times 10^{-5}$ $\mathrm{mol} \cdot \mathrm{L}^{-1}$ 的条件下, 配置系列大环 3 和 $\mathrm{La}^{3+}$ 离子浓度比 不同的反应体系. 待系列体系达反应平衡后，测定系列 体系的吸光度, 将各体系的 $\Delta A$ 对相应的浓度比([3]/ $\left(\left[\mathrm{La}^{3+}\right]+[3]\right)$ 作图(如图 $\left.5 b\right)$. 图 $5 b$ 表明大环 3 与 $\mathrm{La}^{3+}$ 形 成了 $1: 1$ 的配合物，以上两种方法测定所得配位比相 互吻合。

稳定常数测定：按照文献[14]方法所报道的曲线拟 合方法，确定大环化合物 3 与 $\mathrm{La}^{3+}$ 配位反应平衡常数. 平衡常数的计算方程为: $Y=B \times K \times X /(1+K \times X)$, 其中 $X=\left[\mathrm{La}^{3+}\right], Y=\Delta A, B=\Delta \varepsilon \times b$, 经曲线拟合得到 $K$ 即为所 求平衡稳定常数. 实验中, 选择性较高的 $323 \mathrm{~nm}$ 波长处 各平衡体系的 $\Delta A$ 值进行 $K_{\alpha}$ 的计算，曲线拟合结果如图 6. 表明化合物 3 与 $\mathrm{La}^{3+}$ 配位平衡常数为 $7.59 \times 10^{3}$.

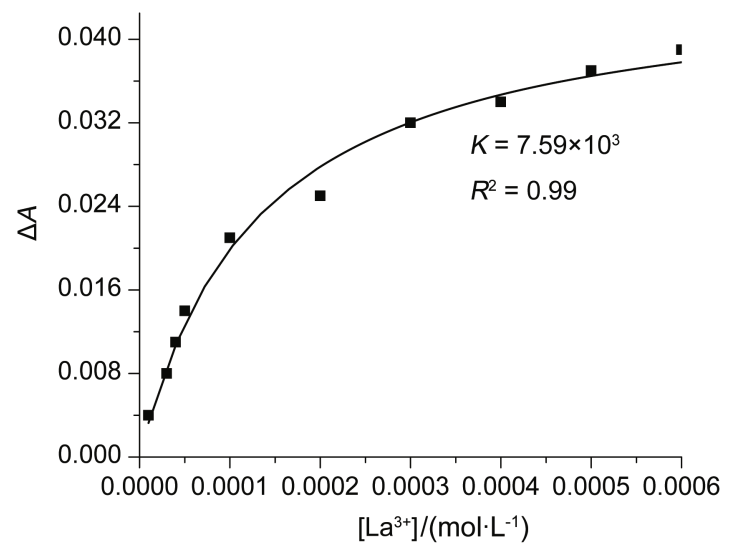

图 6 大环 3 和 $\mathrm{La}^{3+}$ 相互作用体系的平衡常数的曲线拟合 $\left(\lambda_{\max }: 323 \mathrm{~nm}\right)$

Figure 6 Curve fit of the equilibrium constant for the system involving macrocycle 3 with $\mathrm{La}^{3+}$

\section{$2.3{ }^{1} \mathrm{H}$ NMR 和质谱}

前体二醛 1 和二胺 2 进行缩合作用得到 Schiff 碱大 环化合物 $\mathbf{3}$ 后，二醛化合物 $\mathbf{1}$ 在 $\delta 10.447$ 处的醛基质子 峰消失, 大环 3 在 $\delta 8.801$ 处出现两个 $\mathrm{N}=\mathrm{CH}$ 的质子峰, 表明前体 1 中两个醛基均与化合物 2 中胺基发生了全缩 合作用. 同时在 $\delta 4.581$ 处呈现出烷氧链的 4 个亚甲基质 子峰 $\left(\mathrm{OCH}_{2} \mathrm{CH}_{2} \mathrm{O}\right)$. 化合物 3 经过质谱(FABMS)表征 (见辅助材料), 其分子离子峰 $[\mathrm{M}+\mathrm{Na}]^{+} \mathrm{m} / \mathrm{z}$ 为 551.2 , $550.2,549.2$, 数据表明二醛 1 和二胺 2 按照 $1: 1$ 缩合形 成了 $[1+1]$ 的 Schiff碱大环化合物 $\mathbf{3}$, 结果与相应的晶体 结构相吻合.

Schiff 碱大环 3 经 $\mathrm{NaBH}_{4}$ 还原后得大环化合物 4. 在 4 的 ${ }^{1} \mathrm{H}$ NMR 中, 氮原子上的质子 $\delta$ 出现在 3.492 和 2.62 两处，与氮原子相连的 4 个亚甲基质子 $\delta$ 出现在 4.323 处. 烷氧链上的 4 个亚甲基质子 $\delta$ 出现在 4.438 处，与大 环 3 中相应的质子位移值相比, 进一步向高场发生了 位移. 经质谱 MS(ESI)表征, 化合物 4 的分子离子峰 
$\left(\mathrm{M}^{+}+\mathrm{H}\right)$ 为 $m / z$ : 533.3, 532.3, 531.2 (见辅助材料).

\section{$2.4 \mathrm{IR}$ 光谱}

大环化合物 $\mathbf{3}$ 的红外光谱数据表明, 原料二醛 $\mathbf{1}$ 中 $v$ 在 $1688 \mathrm{~cm}^{-1}$ 附近的羰基 $(\mathrm{C}=\mathrm{O})$ 振动峰消失, 在 1621 $\mathrm{cm}^{-1}$ 处出现了很强的 Schiff碱 $\mathrm{C}=\mathrm{N}$ 双键的伸缩振动峰. 大环 3 还原为大环 4 后, 大环 3 中的 $v$ 在 $1621 \mathrm{~cm}^{-1}$ 附 近的 $\mathrm{C}=\mathrm{N}$ 双键的伸缩振动峰消失, 在 $3429 \mathrm{~cm}^{-1}$ 处出 现很强的 $\mathrm{N}-\mathrm{H}$ 伸缩振动峰.

\section{5 阳离子模板合成}

迄今, 文献报道合成席夫碱大环化合物方法主要分 为 4 种, 即高度稀释合成法 ${ }^{[15]}$ 、金属离子模板合成 法 ${ }^{[16,17]}$ 、阴离子模板合成法 ${ }^{[18]}$ 以及酸催化合成法等 ${ }^{[19]}$. 本文在目标大环 3 的合成过程中, 分别采用以上 4 种方 法进行了合成尝试, 但只有采用阳离子模板合成法较为 有效, 其它 3 种合成方法几乎观察不到目标大环的生成, 主要产品为高聚物. 在采用离子模板合成法进行合成尝 试过程中, 我们分别采用了一价或二价的过渡金属离子 进行诱导成环试验, 结果表明, $\mathrm{Ba}^{2+}$ 对诱导合成目标大 环 3 最为有效. 我们推测反应中, 可能是加入 $\mathrm{Ba}^{2+}$ 阳离 子后, $\mathrm{Ba}^{2+}$ 同前体二胺 $\mathbf{2}$ 中配位供体原子氧或氮之间具 有一定的键合作用, 导致前体二胺 2 呈 “钳式” 结构, 从 而有利于它与前体二醛 1 发生缩合成环作用.

\section{3 结论}

本文在合成 $[1+1]$ Schiff 碱大环化合物 3 的过程中, 分别采用了不同方法进行试验, 结果表明, $\mathrm{Ba}^{2+}$ 对诱导 合成目标大环 3 最为有效. 当大环 $\mathbf{3}$ 中的 Schiff 碱 $\mathrm{N}=\mathrm{C}$ 双键被还原为大环 $\mathbf{4}$ 以后, 大环分子构型从折叠 结构转化为扭曲的非折叠结构; 由于环腔尺寸以及环缘 上配位供体原子之间距离和配位取向的差异, 大环 $\mathbf{4}$ 对 考察的系列稀土离子均无明显作用, 仅大环 3 对 $\mathrm{La}^{3+}$ 离 子有选择性键合作用, 其作用的配位比为 $1: 1$, 平衡常 数为 $7.59 \times 10^{3}$.

辅助材料(Supporting Information) 大环 3 和 $\mathbf{4}$ 的 ${ }^{1} \mathrm{H}$
NMR 和 MS 谱图及其晶体学数据. 这些材料可以免费 从本刊网站(http://sioc-journal.cn/)上下载.

\section{References}

[1] Srimurugan, S.; Viswanathan, B.; Varadarajan, T. K.; Varghese, B. Org. Biomol. Chem. 2006, 4, 3044.

[2] Daniel, G. R.; Ludger, A. W. J. Am. Chem. Soc. 2009, 131, 3721.

[3] Wang, M.-X. Chem. Commun. 2008, 4541.

[4] Wang, Z.; Reibenspies, J.; Martell, A. E. Inorg. Chem. 1997, 36, 629.

[5] Ding, C.-X.; Ni, J.; Yang, Y.-H.; Ng, S.-W.; Wang, B.-W.; Xie, Y.-S. CrystEngComm 2012, 14, 7312.

[6] Matthews, R. W.; Mary, M.; Scowen, I. J. J. Chem. Soc, Dalton Trans. 1997, 2861.

[7] Zhang, J.-Q.; Jia, C.-Y. Chin. J. Org. Chem. 2010, 30, 1142 (in Chinese).

(张家强, 贾春阳, 有机化学, 2010, 30, 1142.)

[8] Hu, P.; G, S-Y.; Zhang, Q.-L.; Zhang, Y.-Q.; Zhu, B.-X. Chin. J. Org. Chem. 2013, 33, 325 (in Chinese).

(胡鹏, 郭思颖, 张奇龙, 张云黔, 朱必学, 有机化学, 2013, 33, 325.)

[9] Vigato, P. A.; Tamburini, S.; Bertolo, L. Coord. Chem. Rev. 2007 $251,1311$.

[10] Hou, K.-N.; Yan, H.-L.; Dai, L.; Yuan, H.-H.; Li, X.-X.; Zhao, B. Chin. Chem. Bioeng. 2009, 10, 39 (in Chinese).

(侯可宁, 颜海龙, 代丽, 袁慧慧, 李信信, 赵斌, 化学与生物工 程, 2009, 10, 39.)

[11] Liu, D.-M. M.S. Thesis, Jilin University, Changchun, 2009 (in Chinese). (刘冬美, 硕士论文, 吉林大学, 长春, 2009.)

[12] Sheldrick, G. M. SHELX-97, University of Göttingen, Germany, 1997.

[13] Özkar, S.; Üıkü, D.; Yıldırım, L. T.; Biricik, N.; Gümgüm, B. J. Mol. Struct. 2004, 688, 207.

[14] Sessler, J. L.; Katayev, E.; Pantos, G. D.; Scherbakov, P.; Reshetova, M. D.; Khrustalev, V. N.; Lynch, V. M.; Ustynyuk, Y. A. J. Am. Chem. Soc. 2005, 127, 11442.

[15] Sellamuthu, A.; Kamalraj, S.; Varghese, B.; Johnpaul, M.; Muthusamy, K. Inorg. Chem. 2012, 51, 5580.

[16] Andrew, L. V.; Nathaniel, W. A.; Daryle, H. B.; Joseph, A. H. Inorg. Chem. 1997, 36, 5132.

[17] Yuan, Z.-L.; Zhang, Q.-L.; Zhu, B.-X. Chin. J. Org. Chem. 2006, 26, 1590 (in Chinese). (袁泽利, 张奇龙, 朱必学, 有机化学, 2006, 26, 1590.)

[18] Ravikumar, I.; Pradyut, G. Chem. Soc. Rev. 2012, 41, 3077.

[19] Evgeny, A. K.; Nikolay, V. B.; Victor, N. K.; Yuri, A. U.; Tananaev, I. G. Jonathan, L. S. J. Org. Chem. 2007, 72, 2886.

(Cheng, F.) 


\title{
Supporting Information
}

\section{大环化合物的合成、结构及其对镧(III)离子的识别研究}

\author{
欧 敏 ${ }^{a}$ 邓雅欣 ${ }^{a}$ 王芳芳 ${ }^{a}$ 朱 纯 ${ }^{a}$ \\ 张奇龙 ${ }^{b}$ 朱必学*, \\ ( $a$ 贵州大学大环及超分子化学重点实验室 贵阳 550025) \\ ( ${ }^{b}$ 贵阳医学院化学教研室 贵阳 550004)
}

表 $\mathrm{S} 1$ 大环 3 和 4 的晶体学及结构修正数据

Table S1 Crystal data and structure refinement for macrocycles $\mathbf{3}$ and $\mathbf{4}$

\begin{tabular}{|c|c|c|}
\hline compound & 3 & 4 \\
\hline Empirical & $\mathrm{C}_{34} \mathrm{H}_{26} \mathrm{~N}_{2} \mathrm{O}_{4}$ & $\mathrm{C}_{34} \mathrm{H}_{30} \mathrm{~N}_{2} \mathrm{O}_{4}$ \\
\hline Formula weight & 526.57 & 530.60 \\
\hline Temperature(K) & $293(2)$ & $293(2)$ \\
\hline Crystal system & Orthorhombic & Triclinic \\
\hline Space group & $P$ bca & $P \overline{1}$ \\
\hline$a / \mathrm{nm}$ & $0.9958(4)$ & $1.1297(2)$ \\
\hline$b / \mathrm{nm}$ & $2.0230(8)$ & $1.1802(3)$ \\
\hline$c / \mathrm{nm}$ & $3.0201(12)$ & $1.3375(3)$ \\
\hline$\alpha /\left(^{\circ}\right)$ & 90.00 & $115.68(6)$ \\
\hline$\beta /\left(^{\circ}\right)$ & 90.00 & $96.24(7)$ \\
\hline$\gamma /\left({ }^{\circ}\right)$ & 90.00 & $103.15(6)$ \\
\hline Volume $/ \mathrm{nm}^{3}$ & $6.084(4)$ & $1.5206(5)$ \\
\hline$Z$ & 8 & 2 \\
\hline$D_{\mathrm{c}} /\left(\mathrm{g} \cdot \mathrm{cm}^{-3}\right)$ & 1.150 & 1.159 \\
\hline$\theta$ Range $\left({ }^{\circ}\right)$ & $1 \leq \theta \leq 25.00$ & $0.989 \leq \theta \leq 25.00$ \\
\hline Absorption coefficient $/ \mathrm{mm}^{-1}$ & 0.076 & 0.076 \\
\hline$F(000)$ & 2208 & 560 \\
\hline Crystal size $/ \mathrm{mm}$ & $0.24 \times 0.22 \times 0.21$ & $0.20 \times 0.23 \times 0.19$ \\
\hline Reflections collected & 40483 & 12360 \\
\hline Independent reflections & 5348 & 5291 \\
\hline Observed reflections $[I>2 \sigma(I)]$ & 2691 & 3096 \\
\hline Refinement method & Full-matrix least squares on $F^{2}$ & Full-matrix least squares on $F^{2}$ \\
\hline Number of parameters & 362 & 361 \\
\hline Goodness-of-fit on $F^{2}$ & 1.044 & 1.084 \\
\hline Final $\mathrm{R}$ indices $[I>2 \sigma(I)]$ & $R_{1}=0.0783, \quad \omega R_{2}=0.2168$ & $R_{1}=0.053, \omega R_{2}=0.1468$ \\
\hline Rindices (all data) & $R_{1}=0.1438, \quad \omega R_{2}=0.2475$ & $R_{1}=0.0877, \omega R_{2}=0.166$ \\
\hline Final weighting scheme & $\begin{array}{c}\omega=1 /\left[\sigma^{2}\left(F_{\mathrm{o}}^{2}\right)\right]+(0.0963 P)^{2}+2.3325 P \\
P=\left(F_{\mathrm{o}}^{2}+2 F_{\mathrm{c}}^{2}\right) / 3\end{array}$ & $\begin{array}{c}w=1 /\left[\sigma^{2}\left(F_{\mathrm{o}}^{2}\right)\right]+(0.0734 P)^{2}+0.000 P \\
P=\left(F_{\mathrm{o}}^{2}+2 F_{\mathrm{c}}^{2}\right) / 3\end{array}$ \\
\hline$(\Delta \rho)_{\max } /\left(\mathrm{e} \cdot \mathrm{nm}^{-3}\right)$ & 297 & 133 \\
\hline$(\Delta \rho)_{\min } /\left(\mathrm{e} \cdot \mathrm{nm}^{-3}\right)$ & -242 & -163 \\
\hline
\end{tabular}


表 S2 大环 3 和 4 的部分键长和键角

Table S2 Select bond lengths ( $\mathrm{nm}$ ) and bond angles $\left({ }^{\circ}\right)$ for macrocycles 3 and 4

\begin{tabular}{ll|ll}
\hline $\mathrm{N}(1)=\mathrm{C}(13)$ & $0.1264(5)$ & $\mathrm{N}(2)=\mathrm{C}(28)$ & $0.1260(5)$ \\
$\mathrm{O}(1)-\mathrm{C}(5)$ & $0.1400(5)$ & $\mathrm{O}(3)-\mathrm{C}(21)$ & $0.1447(7)$ \\
$\mathrm{O}(1)-\mathrm{C}(7)$ & $\mathrm{O}(2)-\mathrm{C}(32)$ & $0.1394(5)$ \\
$\mathrm{O}(4)-\mathrm{C}(20)$ & $\mathrm{O}(2)-\mathrm{C}(1)$ & $0.1384(5)$ \\
& $0.1403(6)$ & & \\
$\mathrm{C}(5)-\mathrm{O}(1)-\mathrm{C}(7)$ & $117.1(3)$ & $\mathrm{C}(1)-\mathrm{O}(2)-\mathrm{C}(32)$ & $122.5(3)$ \\
$\mathrm{C}(19)-\mathrm{O}(4)-\mathrm{C}(20)$ & $116.9(5)$ & $\mathrm{C}(21)-\mathrm{O}(3)-\mathrm{C}(22)$ & $122.7(5)$ \\
\hline
\end{tabular}

附: 大环 3 和 4 的 ${ }^{1} \mathrm{H} N \mathrm{NR}$

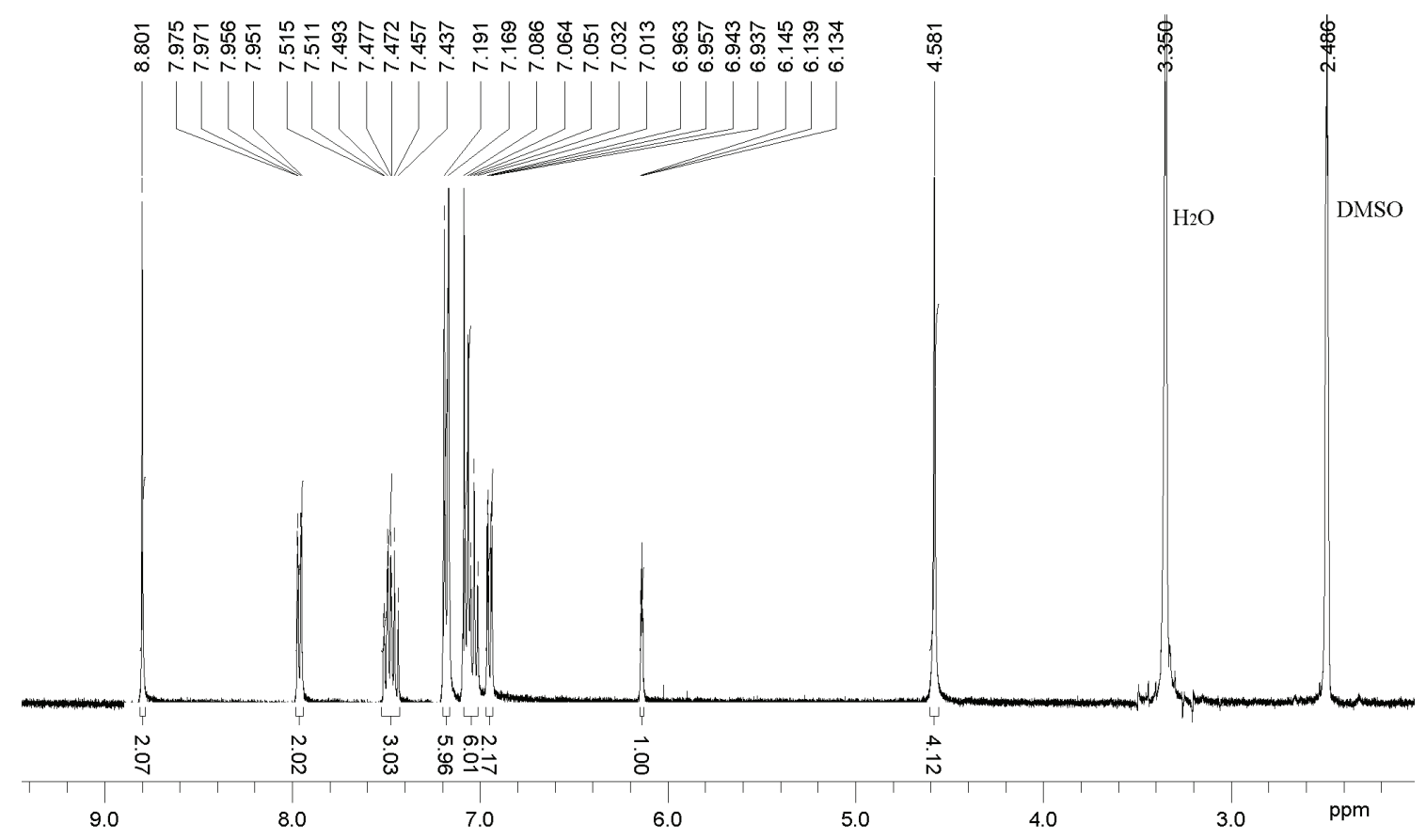

Figure S1 ${ }^{1}$ H NMR for macrocycle 3 


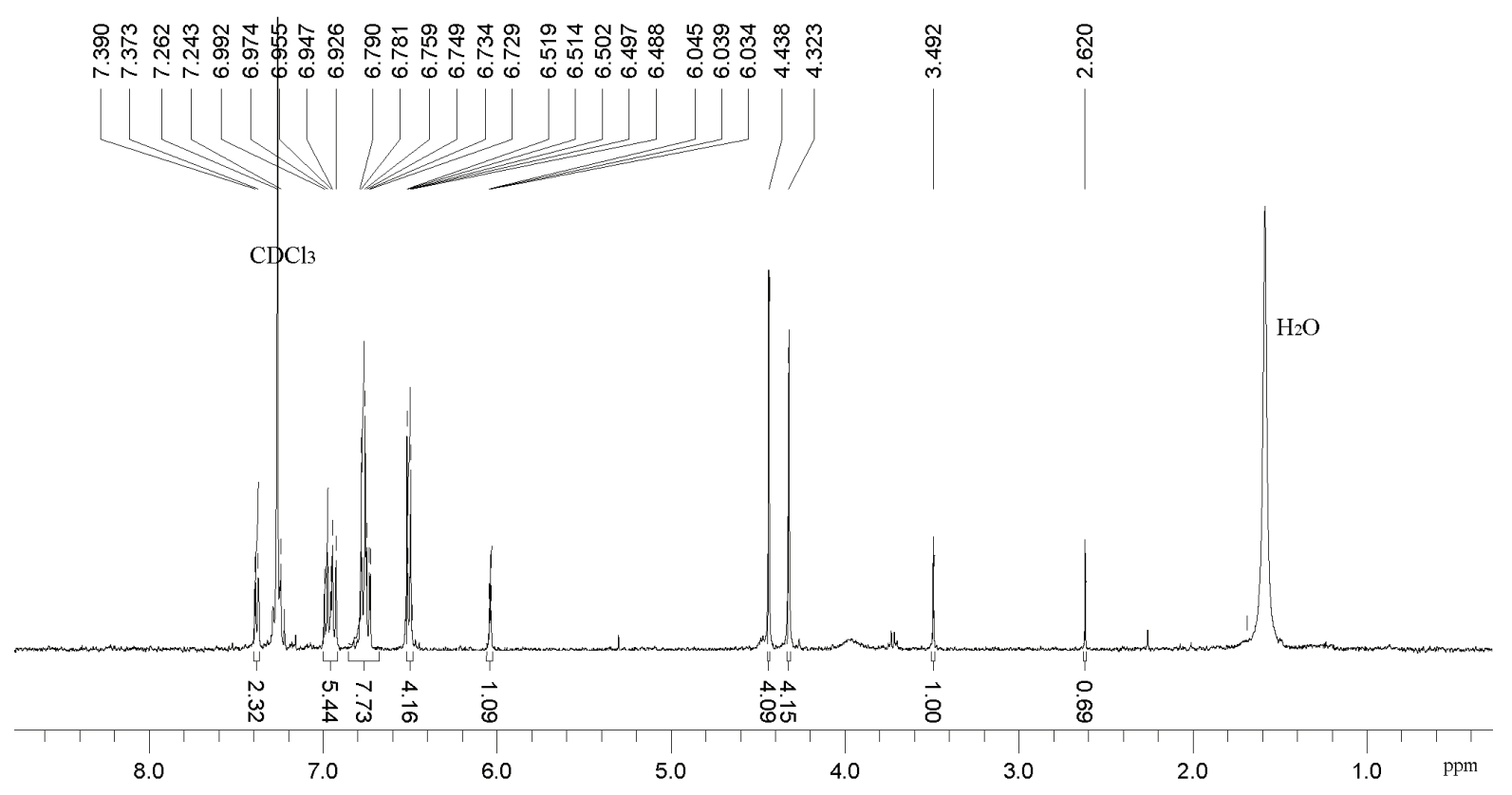

Figure S2 ${ }^{1} \mathrm{H}$ NMR for macrocycle 4

大环 3 和 4 的 MS

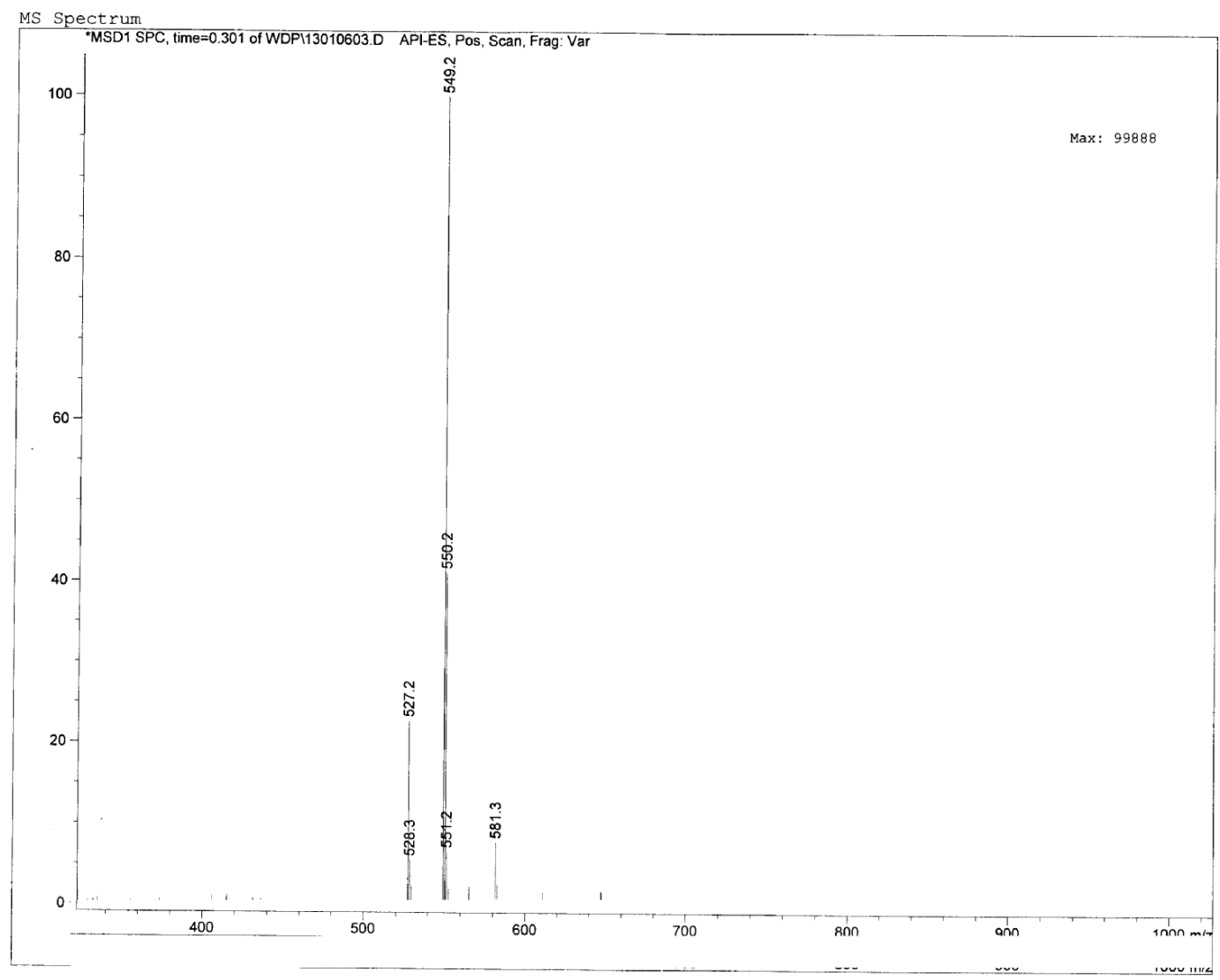

Figure S3 FABMS for macrocycle 3 


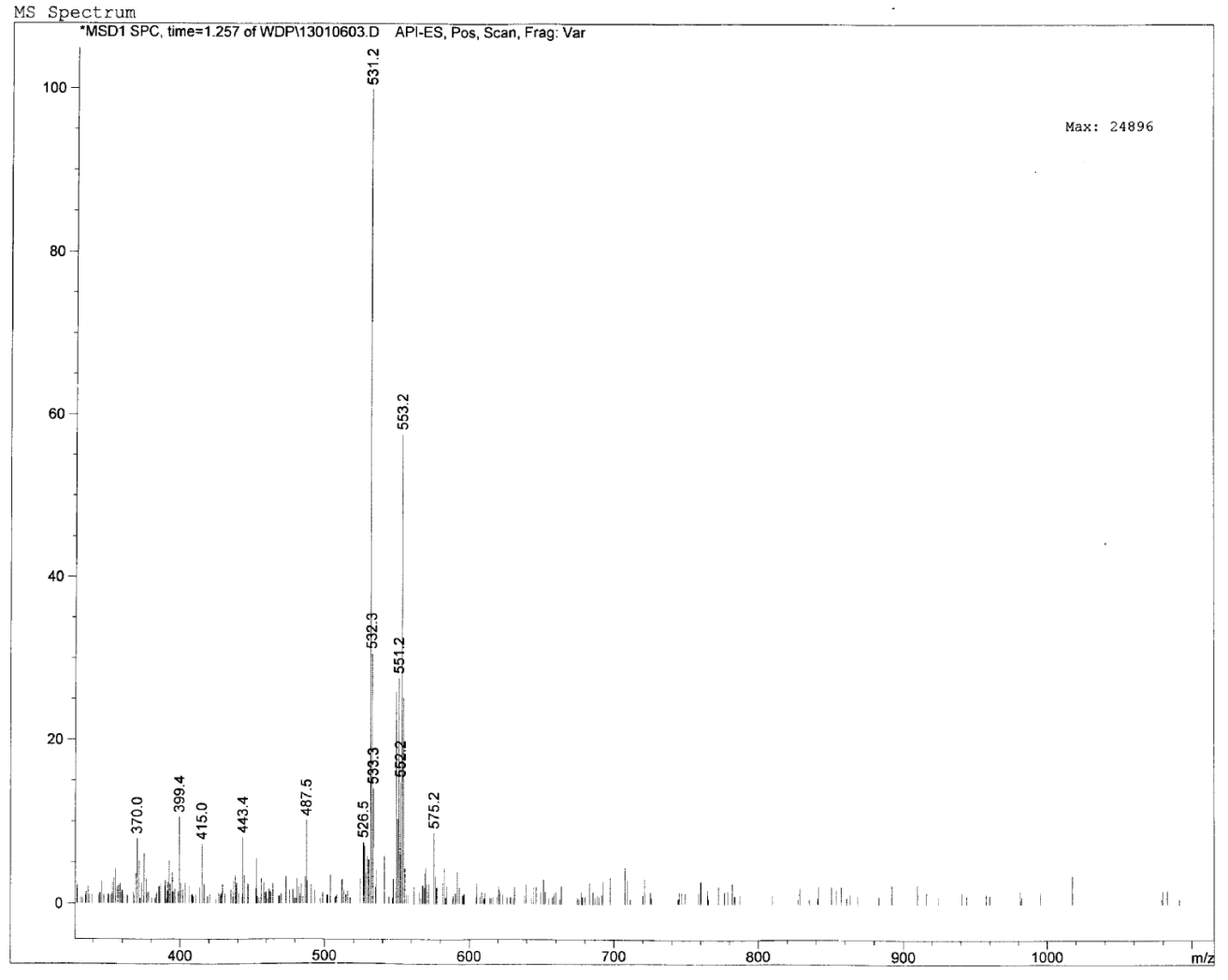

Figure S4 MS(ESI) for macrocycle 4 